\title{
An eigenfunction expansion-variational method in prediction of the transverse thermal conductivity of fiber reinforced composites considering interfacial characteristics
}

\author{
P. Yan ${ }^{a}$, F.L. Chen ${ }^{a}$, C.P. Jiang ${ }^{\mathrm{a}, \mathrm{b}, *}$, F. Song ${ }^{\mathrm{b}}$ \\ a School of Aeronautic Science and Engineering, Beijing University of Aeronautics and Astronautics, 100191 Beijing, China \\ ${ }^{\mathrm{b}}$ State Key Laboratory of Nonlinear Mechanics (LNM), Institute of Mechanics, Chinese Academy of Sciences, 100190 Beijing, China
}

\section{A R T I C L E I N F O}

\section{Article history:}

Received 11 March 2010

Received in revised form 18 June 2010

Accepted 27 June 2010

Available online 1 July 2010

\section{Keywords:}

Fiber reinforced composites

B. Thermal properties

B. Interface

C. Modelling

\begin{abstract}
A B S T R A C T
An eigenfunction expansion-variational method based on a unit cell is developed to deal with the steadystate heat conduction problem of doubly-periodic fiber reinforced composites with interfacial thermal contact resistance or coating. The numerical results show a rapid convergence of the present method. The present solution provides a unified first-order approximation formula of the effective thermal conductivity for different interfacial characteristics and fiber distributions. A comparison with the present high-order results, available experimental data and micromechanical estimations demonstrates that the first-order approximation formula is a good engineering closed-form formula. An engineering equivalent parameter reflecting the overall influence of the thermal conductivities of the matrix and fibers and the interfacial characteristic on the effective thermal conductivity, is found. The equivalent parameter can greatly simplify the complicated relation of the effective thermal conductivity to the internal structure of a composite.
\end{abstract}

(c) 2010 Elsevier Ltd. All rights reserved.

\section{Introduction}

Fiber reinforced composites have been used extensively in many applications from aerospace craft to electric devices, and their effective thermal conductivities have attracted a good deal of attention. A lot of investigations (for example by Hasselman and coworkers [1,2]) show that interfacial gaps exist, which are formed mainly because of the mismatch in thermal expansion coefficients of constituents during the cooling process of manufacture. When the gap thickness is small compared to the mean free path of the gas molecules in the gap, heat conduction is independent of gap thickness. Under this circumstance, thermal contact resistance may be quantified by a heat transfer coefficient, $h$. Besides, it is sometimes desirable to coat the inclusion with a third material to improve the performance of the inclusion in certain physical properties [3]. For either reason, the interfacial characteristics have an important influence on the macroscopic properties of composites. The thermal performance design of a novel composite requires a comprehensive understanding of the effect of the internal structure on the effective thermal conductivity.

\footnotetext{
* Corresponding author at: School of Aeronautic Science and Engineering, Beijing University of Aeronautics and Astronautics, 100191 Beijing, China. Tel.: +86 010 82317508.

E-mail address: jiangchiping@buaa.edu.cn (C.P. Jiang).
}

A lot of researches about the effect of interfacial characteristics on effective thermal conductivities were conducted. Hasselman and Johnson [4] presented a modification of the original theories of Rayleigh and Maxwell considering an interfacial thermal contact resistance, and found that the effective thermal conductivity depends on the dispersion size due to the existence of an interfacial thermal contact resistance. Benveniste and coworkers $[5,6]$ extended the generalized self-consistent method and the MoriTanaka method to solve the problems of the interfacial thermal contact resistance and coated fibers. Lee et al. [7] obtained the effective thermal conductivity of composites with interfacial contact conductance or multi-layer inclusions by using the generalized self-consistent method. Böhm and Nogales [8] presented a Mori-Tanaka scheme for modeling the overall thermal conduction behavior of composites containing reinforcements with interfacial resistances and prescribed size distributions. The Mori-Tanaka method and generalized self-consistent method are two different micromechanics methods, and generally give different results, but they and Hasselman's method [4] give the same formula of effective thermal conductivity for the circularsection fiber reinforced composites discussed in the present paper. Zou et al. [9] derived an analytical expression for transverse thermal conductivities of fiber composites with thermal barrier based on the electrical analogy technique and on the cylindrical filament-square packing array unit cell model. 
The above-mentioned works presented closed-form formulae for effective thermal conductivities by treating the inclusion interactions either approximately or in a statistical sense. Along another lines, many works take periodic composite models to consider exact inclusion interactions. The periodic composite models provide useful limiting values of interacting inclusions from entirely disorder (random) to order. At the same time, the so-called composite design is generally a design of periodically distributed unit cells of a composite [10]. Gu and Liu [11] discussed the thermal conductivity of periodic composite media with contact resistance by applying a generalized Rayleigh identity. Lu and Lin [12] studied the effect of interfacial characteristics on the effective thermal conductivity of two-dimensional periodic composites by using a boundary collocation scheme. These two works are both based on series expansion approaches of real functions, in which the interfacial conditions between inclusions and the matrix are satisfied in advance, and then the periodicity conditions are used to determine the remaining expansion coefficients. In contrast with the finite element methods $[13,14]$, the two works presented good semi-analytical methods.

From existing researches, it is seen that the effective thermal conductivity of a composite is related to complicated micromechanical parameters, such as the thermal conductivities of constituents, interfacial characteristics and distribution of inclusions. Apparently, unified and convenient engineering closed-form formulae of effective thermal conductivities considering the periodic microstructures and different interfacial characteristics are highly desirable for the design of composites.

First, the present work is devoted to develop an eigenfunction expansion-variational method [15] in prediction of the transverse thermal conductivity of fiber reinforced composites considering interfacial characteristics, in which the eigenfunction expansion approach is based on complex functions and the periodicity conditions are dealt with by using a variational method. Then the emphasis is laid on the development of the efficient and convenient engineering closed-form formula as well as the extraction of the engineering equivalent parameter, which can greatly simplify the complicated relation of the effective thermal conductivity to the internal structure of a composite.

\section{Description of a unit cell and the generalized variational functional}

\subsection{Basic equations}

For the problem of steady-state heat conduction with no internal generation, the heat flux $\mathbf{q}$, temperature field $T$ and temperature gradient $\mathbf{H}$ satisfy the following three equations [16]:

Fourier's law : $\mathbf{q}=-\mathbf{k} \cdot \mathbf{H}$

Temperature gradient : $\mathbf{H}=\nabla T$

Equilibrium equation : $\nabla \cdot \mathbf{q}=0$

where $\mathbf{k}$ is the thermal conductivity tensor. For a transversely isotropic material, the in-plane temperature field satisfies Laplace's equation [16]

$\frac{\partial^{2} T}{\partial x_{1}^{2}}+\frac{\partial^{2} T}{\partial x_{2}^{2}}=0$

\subsection{Description of a unit cell}

A composite with a general doubly-periodic array of circular cross-section fibers is shown in Fig. 1, where $\mathbf{d}_{1}$ and $\mathbf{d}_{2}$ denote two fundamental periods. For the same periodic structure, different unit cells can be selected, for example, the two unit cells in
Fig. 1b and c. From the doubly-periodic distribution of unit cells, the boundaries of a unit cell can be divided into $\partial V_{j}^{+}$and $\partial V_{j}^{-}$in pairs, where $j=1,2,3$ for Fig. $1 \mathrm{~b}$ and $j=1,2$ for Fig. 1c. By a proper translation $\mathbf{p}^{j}$, the boundary $\partial V_{j}^{-}$will coincide with the boundary $\partial V_{j}^{+}$, where $\mathbf{p}^{1}=\mathbf{d}_{1}-\mathbf{d}_{2}, \mathbf{p}^{2}=\mathbf{d}_{1}, \mathbf{p}^{3}=\mathbf{d}_{2}$ for Fig. $1 \mathrm{~b}$ and $\mathbf{p}^{1}=\mathbf{d}_{1}$, $\mathbf{p}^{2}=\mathbf{d}_{2}$ for Fig. 1c. For steady-state heat conduction, the temperature field $T(\mathbf{x})$ is quasi-periodic and the heat flux field $\mathbf{q}(\mathbf{x})$ is periodic, thus the corresponding temperatures $T$ and boundary heat flux $q(=\mathbf{q} \cdot \mathbf{n})$ on $\partial V_{j}^{+}$and $\partial V_{j}^{-}$satisfy the following periodic boundary conditions:

$\left\{\begin{array}{l}T^{j+}-T^{j-}=\langle\mathbf{H}\rangle \cdot \mathbf{p}^{j} \\ q^{j+}+q^{j-}=0\end{array}\right.$

where $\mathbf{n}$ denotes the unit normal vector on the boundaries, $\langle\mathbf{H}\rangle$ denotes the average temperature gradient within a unit cell and is the same for all unit cells.

\subsection{Generalized variational functional for a unit cell}

The energy functional for heat conduction can been defined as

$\Pi=\int_{V} \frac{1}{2} \mathbf{q} \cdot \mathbf{H} d V$

where $V$ is the volume of a unit cell. By using the Lagrangian multiplier method, the periodic boundary conditions (2) of a unit cell can be incorporated in the functional (3):

$\Pi=\int_{V} \frac{1}{2} \mathbf{q} \cdot \mathbf{H} d V-\sum_{j} \int_{\partial V_{j}^{+}} q^{j+} \cdot\left(T^{j+}-T^{j-}-\langle\mathbf{H}\rangle \cdot \mathbf{p}^{j}\right) d S$

The stationary condition of the functional (4) yields

$$
\begin{aligned}
& \sum_{j} \int_{\partial V_{j}^{+}} \delta q^{j+}\left(T^{j+}-T^{j-}\right) d S-\sum_{j} \int_{\partial V_{j}^{+}}\left(q^{j+}+q^{j-}\right) \delta T^{j-} d S \\
& =\sum_{j} \int_{\partial V_{j}^{+}} \delta q^{j+}\langle\mathbf{H}\rangle \cdot \mathbf{p}^{j} d S
\end{aligned}
$$

which will be used to develop an eigenfunction expansion-variational method based on a unit cell.

\section{Eigenfunction expansions of complex potentials}

\subsection{Complex potential}

From Eqs. (1a)-(1d), the temperature $T$, heat flux components $\left\{q_{1}, q_{2}\right\}$ and heat transfer rate $\Phi$ can be formulated by a complex potential $\omega(z)$ :

$q_{1}-\mathrm{i} q_{2}=-k \omega^{\prime}(z)$

$T=\frac{1}{2}[\omega(z)+\overline{\omega(z)}]$

$\Phi=\frac{-k}{2 \mathrm{i}}[\omega(z)-\overline{\omega(z)}]_{\mathrm{A}}^{\mathrm{B}}$

where $z=x_{1}+i x_{2}$ is a complex variable, the over bar denotes the complex conjugate, the prime denotes the derivative with respect to $z,[\cdot]_{A}^{B}$ denotes the difference of the values of the bracketed function from point $\mathrm{A}$ to point $\mathrm{B}$, and $k$ is the thermal conductivity.

\subsection{Eigenfunction expansions in the coating case}

Now derive eigenfunction expansions of the complex potentials in a unit cell and first consider the case of coating. Without loss of generality, take the unit cell in Fig. 1b as an example, which is shown in Fig. 2a where $R$ and $R_{1}$ are the internal and external radii of the coating, respectively. 


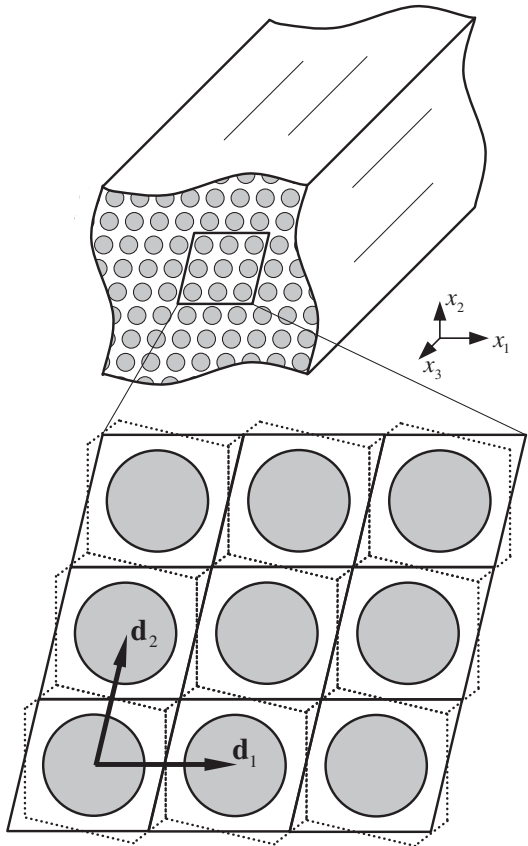

(a)

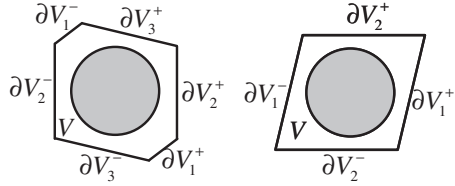

(b)

(c)

Fig. 1. Doubly-periodic fiber reinforced composite and its two kinds of unit cells

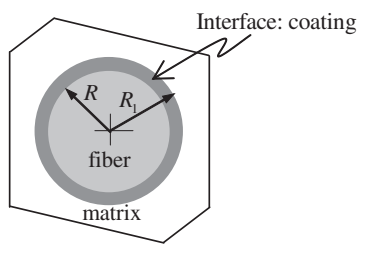

(a)

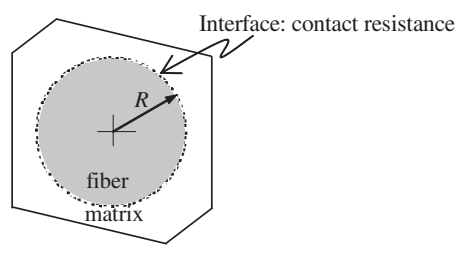

(b)
Fig. 2. A unit cell with a fiber: (a) coating interface; (b) thermal contact resistance interface.

The complex potential $\omega_{\mathrm{f}}(z)$ in the fiber region can be expanded into a Taylor series, $\omega_{\mathrm{c}}(z)$ in the coating region and $\omega_{\mathrm{m}}(z)$ in the matrix region can be expanded into Laurent series, respectively:

$\omega_{\mathrm{f}}(z)=\sum_{n=1}^{\infty} E_{n} z^{2 n-1}$

$\omega_{\mathrm{c}}(z)=\sum_{n=1}^{\infty} P_{n} z^{-(2 n-1)}+\sum_{n=1}^{\infty} Q_{n} z^{2 n-1}$

$\omega_{\mathrm{m}}(z)=\sum_{n=1}^{\infty} G_{n} z^{-(2 n-1)}+\sum_{n=1}^{\infty} F_{n} z^{2 n-1}$

where $E_{n}, P_{n}, Q_{n}, G_{n}$ and $F_{n}$ are complex coefficients. Due to the centrosymmetry of the unit cell, only odd terms in Eqs. (7)-(9) remain.

The continuity conditions of the heat transfer rate $\Phi$ and temperature $T$ across the fiber-coating and coating-matrix interfaces can be written as

$\Phi_{\mathrm{f}}=\Phi_{\mathrm{c}}, T_{\mathrm{f}}=T_{\mathrm{c}} \quad$ at $|z|=R$

$\Phi_{\mathrm{c}}=\Phi_{\mathrm{m}}, T_{\mathrm{c}}=T_{\mathrm{m}} \quad$ at $|z|=R_{1}$

where the subscripts $\mathrm{f}, \mathrm{c}$ and $\mathrm{m}$ refer to the fiber, coating and matrix, respectively. The interfacial conditions (Eqs. (10) and (11)) can provide four sets of equations with respect to five sets of unknown complex coefficients $E_{n}, P_{n}, Q_{n}, G_{n}$ and $F_{n}$. Only a set of independent unknown complex coefficients (choose $F_{n}$ ) remains. It will be seen that the estimation of the effective conductivity requires only $\omega_{\mathrm{m}}(z)$. Substituting Eqs. (7)-(9) into Eqs. (6a)-(6c) and then into Eqs. (10) and (11), one obtains the relation between $G_{n}$ and $F_{n}$ :

$G_{n}=\frac{\eta_{\mathrm{cm}}+\eta_{\mathrm{fc}}(1+\xi)^{2-4 n}}{1+\eta_{\mathrm{cm}} \eta_{\mathrm{fc}}(1+\xi)^{2-4 n}} R_{1}^{4 n-2} \bar{F}_{n}$

where

$$
\begin{aligned}
\eta_{\mathrm{fc}} & =\left(k_{\mathrm{c}}-k_{\mathrm{f}}\right) /\left(k_{\mathrm{c}}+k_{\mathrm{f}}\right), \quad \eta_{\mathrm{cm}}=\left(k_{\mathrm{m}}-k_{\mathrm{c}}\right) /\left(k_{\mathrm{m}}+k_{\mathrm{c}}\right), \\
\xi & =\left(R_{1}-R\right) / R
\end{aligned}
$$

and $\xi$ is called as the relative thickness of the coating.

\subsection{Eigenfunction expansions in the contact resistance case}

A unit cell with a contact resistance at the fiber-matrix interface is shown in Fig. 2b. The expansions of $\omega_{\mathrm{f}}(z)$ and $\omega_{\mathrm{m}}(z)$ are the same as those in Eqs. (7) and (9), respectively. For the contact resistance problem, the continuity condition of the heat transfer rate $\Phi$ and the discontinuity condition of the temperature can be written as

$\Phi_{\mathrm{f}}=\Phi_{\mathrm{m}}, q_{\mathrm{f}}=q_{\mathrm{m}}=-h\left(T_{\mathrm{f}}-T_{\mathrm{m}}\right) \quad$ at $|z|=R$

where $h$ is the thermal contact conductance on the interface. Substituting Eqs. (7) and (9) into Eqs. (6a)-(6c) and then into Eq. (14), one obtains:

$G_{n}=\frac{\eta_{\mathrm{fm}}+(2 n-1) \beta\left(1-\eta_{\mathrm{fm}}\right)}{1+(2 n-1) \beta\left(1-\eta_{\mathrm{fm}}\right)} R^{4 n-2} \bar{F}_{n}$

where

$\eta_{\mathrm{fm}}=\left(k_{\mathrm{m}}-k_{\mathrm{f}}\right) /\left(k_{\mathrm{m}}+k_{\mathrm{f}}\right), \quad \beta=k_{\mathrm{m}} /(2 h R)=1 /(2 B i)$

and $B i$ is the Biot number. It is found from Eq. (15) that the combined effect of the thermal contact conductance $h$ and the fiber radius $R$ is characterized by a composite parameter $\beta$. 


\subsection{Unified expression of the eigenfunction expansions}

It is seen that for the problems of coating and contact resistance, Eqs. (12) and (15) can be cast into a unified expression:

$G_{n}=\eta_{n} \bar{F}_{n}$

where

$\eta_{n}= \begin{cases}\frac{\eta_{\mathrm{fm}}+(2 n-1) \beta\left(1-\eta_{\mathrm{fm}}\right)}{1+(2 n-1) \beta\left(1-\eta_{\mathrm{fm}}\right)} R^{4 n-2} & \text { contact resistance } \\ \frac{\left.\eta_{\mathrm{cm}}+\eta_{\mathrm{fc}}(1+\xi)\right)^{2-4 n}}{1+\eta_{\mathrm{cm}} \eta_{\mathrm{fc}}(1+\xi)^{2-4 n}} R_{1}^{4 n-2} & \text { coating }\end{cases}$

So for the two cases of coating and contact resistance, the eigenfunction expansion of the complex potential in the matrix region can be written as a unified expression:

$\omega_{\mathrm{m}}(z)=\sum_{n=1}^{\infty} \eta_{n} \bar{F}_{n} z^{-(2 n-1)}+\sum_{n=1}^{\infty} F_{n} z^{2 n-1}$

The eigenfunction expansion in Eq. (19) satisfies the equilibrium equations and the fiber-matrix interface conditions. The remaining work is the determination of the set of independent unknown coefficients $F_{n}$, which can be completed by using the stationary conditions (Eq. (5)).

\section{Determination of the unknown coefficients in eigenfunction expansions}

Substituting Eq. (19) into Eqs. (6a)-(6c), and taking an appropriate truncation of the expansions, the heat flux, temperature and heat transfer rate can be expressed as follows

$q_{i}=\sum_{n=1}^{2 N} X_{n} q_{i}^{(n)}, \quad T=\sum_{n=1}^{2 N} X_{n} T^{(n)}, \quad \Phi=\sum_{n=1}^{2 N} X_{n} \Phi^{(n)} \quad i=1,2$

where

$X_{n}= \begin{cases}\eta_{n} \bar{F}_{n} & 1 \leqslant n \leqslant N \\ F_{n-N} & N+1 \leqslant n \leqslant 2 N\end{cases}$

the detailed expressions of $q_{i}^{(n)}, T^{(n)}$ and $\Phi^{(n)}$ can be obtained from Eqs. (6a)-(6c) and (19).

The substitution of Eq. (20) into the Eq. (5)) yields the following set of linear algebraic equations:

$\sum_{n=1}^{2 N} A_{m n} X_{n}=B_{m} \quad m=1,2, \ldots, 2 N$

where

$A_{m n}=\sum_{j} \int_{\partial V_{j}^{+}} \mathbf{n}^{j+} \cdot \mathbf{q}_{(m)}^{j+}\left(T_{(n)}^{j+}-T_{(n)}^{j-}\right) d S-\sum_{j} \int_{\partial V_{j}^{+}} \mathbf{n}^{j-} \cdot\left(\mathbf{q}_{(n)}^{j-}-\mathbf{q}_{(n)}^{j+}\right) T_{(m)}^{j-} d S(22 \mathrm{~b})$

$B_{m}=\sum_{j} \int_{\partial V_{j}^{+}}\left(\mathbf{n}^{j+} \cdot \mathbf{q}_{(m)}^{j+}\right)\left(\langle\mathbf{H}\rangle \cdot \mathbf{p}^{j}\right) d S=\sum_{j}\left[\Phi_{(m)}^{j+}\langle\mathbf{H}\rangle \cdot \mathbf{p}^{j}\right]$

$\mathbf{q}_{(m)}^{j+}, T_{(n)}^{j+}$ and $\Phi_{(m)}^{j+}$ denote the values of $q_{i}^{(m)}, T^{(n)}$ and $\Phi^{(m)}$ on $\partial V_{j}^{+}$, and the superscript " $j-$ " refers to $\partial V_{j}^{-}$.

After determining the unknown coefficients, the heat flux and temperature fields are obtained from Eq. (20).

\section{Effective thermal conductivities}

The effective thermal conductivities $k_{i j}^{\mathrm{e}}$ are determined with the aid of the average field theory:

$\left\langle q_{i}\right\rangle=-k_{i j}^{\mathrm{e}}\left\langle H_{j}\right\rangle$

where $\left\langle q_{i}\right\rangle$ is the average heat flux in the unit cell. Due to its symmetry, the transverse thermal conductivity tensor has three independent components: $k_{11}^{\mathrm{e}}, k_{22}^{\mathrm{e}}, k_{12}^{\mathrm{e}}=k_{21}^{\mathrm{e}}$. For a composite with a square or hexagonal array of fibers $k_{12}^{\mathrm{e}}=k_{21}^{\mathrm{e}}=0, k_{11}^{\mathrm{e}}=k_{22}^{\mathrm{e}}=k_{\mathrm{e}}$.

From the present series solution, two products of practical importance are obtained. One is that the first-order approximation of the effective thermal conductivity can be written into a unified formula for three cases of perfect interface, thermal contact resistance interface and coating phase between fibers and the matrix, and for the square and hexagonal arrays of fibers. In fact, taking the series term number $N=1$, one obtains

$\frac{k_{\mathrm{e}}}{k_{\mathrm{m}}}=\frac{(\pi-\alpha \eta \lambda)^{2}}{2 \pi^{2}(1+\eta \lambda)-(\pi+\alpha \eta \lambda)^{2}}$

where $\lambda$ is the fiber volume fraction in the cases of the perfect interface and contact resistance interface, or a combined volume fraction of fibers and coating in the case of coated fibers; $\eta$ is a composite parameter characterizing the constituent properties and interfacial characteristics,

$\eta= \begin{cases}\frac{\left(k_{\mathrm{m}}-k_{\mathrm{f}}\right)}{\left(k_{\mathrm{m}}+k_{\mathrm{f}}\right)} & \text { Perfect interface } \\ \frac{\eta_{\mathrm{fm}}+\beta\left(1-\eta_{\mathrm{fm}}\right)}{1+\beta\left(1-\eta_{\mathrm{fm}}\right)} & \text { Contact resistance interface } \\ \frac{\eta_{\mathrm{cm}}+\eta_{\mathrm{fc}}(1+\xi)^{-2}}{1+\eta_{\mathrm{cm}} \eta_{\mathrm{fc}}(1+\xi)^{-2}} & \text { Coating }\end{cases}$

$\eta_{\mathrm{fm}}, \eta_{\mathrm{fc}}, \eta_{\mathrm{cm}}, \beta$ and $\xi$ are given in Eqs. (13) and (16), and $\alpha$ is related to distribution of fibers:

$\alpha= \begin{cases}2 & \text { Square array } \\ 3 \sqrt{3} / 2 & \text { Hexagonal array }\end{cases}$

It is seen that $-1 \leqslant \eta \leqslant 1$ for a composite. The numerical results in the next section will show that Eq. (24a) is a good unified engineering closed-form formula of the effective thermal conductivity.

It is interesting to note that in existing references, the problems of perfect interface, contact resistance interface and coated fibers were separately investigated by using the generalized self-consistent method [5-7], and different formulae were presented. We find that those unrelated formulae can also be cast into a unified expression in terms of $\eta$ :

$\frac{k_{\mathrm{e}}}{k_{\mathrm{m}}}=1-\frac{2 \eta \lambda}{1+\eta \lambda}$

A comparison of Eq. (24a) with Eq. (25) shows that the present first-order approximation formula of the effective conductivity can reflect the effect of fiber distribution, so can be regarded as an improvement of the generalized self-consistent formula.

The second product of the present work is to find an engineering equivalent parameter $\eta$ (see Eq. (24b)), which can replace the fiber and matrix thermal conductivities and the interfacial parameter with a good engineering accuracy. Such an engineering equivalent parameter can greatly simplify the relation of the effective thermal conductivity to the composite internal structure, which is helpful to a comprehensive understanding of thermal properties of composites as well as to design of novel composites

If $\eta=0$, that is $\beta=\eta_{\mathrm{fm}} /\left(-1+\eta_{\mathrm{fm}}\right)$ for a contact resistance problem and $\xi=\sqrt{-\eta_{\mathrm{fc}} / \eta_{\mathrm{cm}}}-1$ for a coated fiber problem in Eq. (24b), it is seen from Eq. (24a) that $k_{\mathrm{e}} / k_{\mathrm{m}}=1$, i.e., the effect of the reinforcements is exactly balanced out by that of the interfaces whose properties meeting some conditions, such that no effect of reinforcement can be realized. Such critical interfacial characteristics were also found by the existing researches $[17,18]$ for spherical particulate or cylindrical fiber reinforced composites, and are verified by the following high-order calculations (see Tables 1 and 2). Two critical interfacial parameters

$\beta_{0}=\eta_{\mathrm{fm}} /\left(-1+\eta_{\mathrm{fm}}\right), \quad \xi_{0}=\sqrt{-\eta_{\mathrm{fc}} / \eta_{\mathrm{cm}}}-1$

are defined, which will be used in the following calculations. 
Table 1

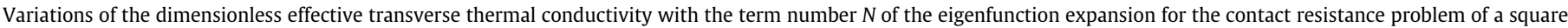
array fibers, where $k_{\mathrm{f}} / k_{\mathrm{m}}=1000, \beta_{0}=0.4995, \lambda=0.7$.

\begin{tabular}{|c|c|c|c|c|c|c|c|}
\hline \multirow[t]{2}{*}{$N$} & \multicolumn{7}{|l|}{$\underline{k_{\mathrm{e}}} / k_{\mathrm{m}}$} \\
\hline & $\begin{array}{l}\eta=-0.998 \\
\frac{\beta}{\beta_{0}}=0\end{array}$ & $\begin{array}{l}\eta=-0.980 \\
\frac{\beta}{\beta_{0}}=0.009\end{array}$ & $\begin{array}{l}\eta=-0.818 \\
\frac{\beta}{\beta_{0}}=0.099\end{array}$ & $\begin{array}{l}\eta=0 \\
\frac{\beta}{\beta_{0}}=1\end{array}$ & $\begin{array}{l}\eta=0.818 \\
\frac{\beta}{\beta_{0}}=10.009\end{array}$ & $\begin{array}{l}\eta=0.980 \\
\frac{\beta}{\beta_{0}}=100.1\end{array}$ & $\begin{array}{l}\eta=0.998 \\
\frac{\beta}{\beta_{0}}=1001\end{array}$ \\
\hline 1 & 7.0879 & 6.6479 & 4.1306 & 1 & 0.31460 & 0.24252 & 0.23537 \\
\hline 3 & 7.2506 & 6.6764 & 3.9189 & 1 & 0.24946 & 0.15625 & 0.14667 \\
\hline 5 & 7.3412 & 6.7286 & 3.9058 & 1 & 0.24239 & 0.14672 & 0.13686 \\
\hline 7 & 7.3638 & 6.7425 & 3.9061 & 1 & 0.24171 & 0.14573 & 0.13584 \\
\hline 9 & 7.3675 & 6.7445 & 3.9061 & 1 & 0.24161 & 0.14558 & 0.13568 \\
\hline 11 & 7.3681 & 6.7448 & 3.9061 & 1 & 0.24159 & 0.14558 & 0.13565 \\
\hline
\end{tabular}

Table 2

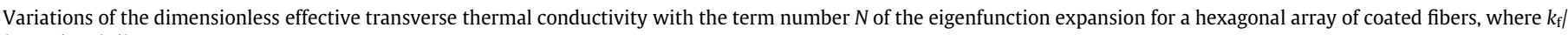
$k_{\mathrm{m}}=1 / 10, k_{\mathrm{c}} / k_{\mathrm{m}}=100, \xi_{0}=0.009, \lambda=0.8$.

\begin{tabular}{|c|c|c|c|c|c|c|c|}
\hline \multirow[t]{2}{*}{$\mathrm{N}$} & \multicolumn{7}{|l|}{$k_{\mathrm{e}} / k_{\mathrm{m}}$} \\
\hline & $\begin{array}{l}\eta=-0.980 \\
\frac{\xi}{\xi_{0}}=\infty\end{array}$ & $\begin{array}{l}\eta=-0.939 \\
\frac{\xi}{\xi_{0}}=42.70\end{array}$ & $\begin{array}{l}\eta=-0.818 \\
\frac{\xi}{\xi_{0}}=11.55\end{array}$ & $\begin{array}{l}\eta=-0.519 \\
\frac{\xi}{\xi_{0}}=3.44\end{array}$ & $\begin{array}{l}\eta=0 \\
\frac{\xi}{\xi_{0}}=1\end{array}$ & $\begin{array}{l}\eta=0.519 \\
\frac{\xi}{\xi_{0}}=0.239\end{array}$ & $\begin{array}{l}\eta=0.818 \\
\frac{\xi}{\xi_{0}}=0\end{array}$ \\
\hline 1 & 8.8195 & 7.4131 & 4.9440 & 2.4463 & 1 & 0.41995 & 0.22540 \\
\hline 3 & 8.7409 & 7.3554 & 4.9150 & 2.4390 & 1 & 0.41317 & 0.20147 \\
\hline 5 & 9.0113 & 7.5343 & 4.9776 & 2.4456 & 1 & 0.41319 & 0.20225 \\
\hline 7 & 9.0279 & 7.5453 & 4.9814 & 2.4460 & 1 & 0.41319 & 0.20207 \\
\hline 9 & 9.0278 & 7.5452 & 4.9814 & 2.4460 & 1 & 0.41319 & 0.20207 \\
\hline 11 & 9.0284 & 7.5456 & 4.9815 & 2.4460 & 1 & 0.41319 & 0.20207 \\
\hline
\end{tabular}

\section{Numerical examples and discussions}

\subsection{Convergence analysis}

Consider the contact resistance problem. Take the fiber-tomatrix conductivity ratio $k_{\mathrm{f}} / k_{\mathrm{m}}=1000$, the critical contact resistance parameter $\beta_{0}=0.4995, \quad \eta= \pm 0.998, \pm 0.980, \pm 0.818,0$ (it is noted that for a practical composite, $-1<\eta<1$ ). Then consider a coated fiber problem. Take $k_{\mathrm{f}} / k_{\mathrm{m}}=1 / 10, k_{\mathrm{c}} / k_{\mathrm{m}}=100, \eta=-0.998$, $-0.939,0, \pm 0.519, \pm 0.818$, the critical relative coating thickness $\xi_{0}=0.009$. The variations of the dimensionless effective transverse thermal conductivity $k_{\mathrm{e}} / k_{\mathrm{m}}$ with the term number $N$ of the eigenfunction expansion are listed in Table 1 for a square array $(\lambda=0.7)$ and in Table 2 for a hexagonal array $(\lambda=0.8)$, respectively.

From Tables 1 and 2, a rapid convergence is observed. It is also shown that the relative error of the first-order approximation formula to the high-order numerical result is less than $5 \%$ as $-0.998<\eta<0$ (corresponding to $1<k_{\mathrm{f}} / k_{\mathrm{m}}<1000$ in the case of perfect interface), and $\lambda<0.7$ for a square array and $\lambda<0.8$ for a hexagonal array. Further calculations show that the results of the first-order approximation formula are still reasonable to some extent for extreme cases of $\eta$ and $\lambda$. Therefore, the present first-order approximation expression can serve as a good engineering closedform formula.

\subsection{Comparison with existing theoretical predictions and experimental data}

In this subsection, a comparison is made with generalized selfconsistent predictions and experimental data. Because of difficulty in measuring, to our best knowledge, no experimental data about the interfacial thermal contact resistance were reported. In the following comparison, the interfacial parameter $\beta$ is conversely determined by experimental data.

Thornburg and Pears [19] conducted experiments on the transverse thermal conductivity of glass reinforced plastics $\left(k_{\mathrm{f}} / k_{\mathrm{m}}=4.4\right)$ and graphite fiber reinforced plastics $\left(k_{\mathrm{f}} / k_{\mathrm{m}}=666\right)$. A comparison of the present first-order approximation formula with the general- ized self-consistent method (GSCM) and the experimental data is depicted in Fig. 3a and b, where EEVM=eigenfunction expansion-variational method and term number $N=1$.

It is seen that the present estimations of the effective thermal conductivity are higher than those by the GSCM. The results for the hexagonal array (HEX) very approach those by the GSCM and the results for the square array (SQU) are larger than them. Such a phenomenon is not surprising. The thermal conductivity of fibers is much higher than that of the matrix. So the shorter the path of heat flow through the matrix is, the higher the effective thermal conductivity of the composite is. The adjacent fibers in a square array are closer together than those in a hexagonal array for the same fiber volume fraction, which results in more fluent heat flow between the higher conductivity phases (the fibers). For a square array, when the fiber volume fraction $\lambda$ approaches 0.785 , the fibers are in contact with each other, which yields a fluent heat flow path. For a hexagonal array, the fibers are not in contact with each other until $\lambda=0.907$. Therefore, the effective thermal conductivity for the square array is higher than that for the hexagonal array. The GSCM is based on an imaginary idealized even distribution of fibers, and the hexagonal array is the closest to the idealized even distribution. From Fig. 3a and b, it is also seen that the present results are in reasonable agreement with the experimental data. Because no experimental data about the interfacial thermal contact resistance are available, in Fig. 3a the values of $\beta$ is conversely determined by experimental data. In Fig. 3b, the experimental data of the effective thermal conductivity are slightly larger than the theoretical estimations. Because of a lack of the interfacial characteristic data, further experimental investigations are still highly desirable.

\subsection{A discussion on the engineering equivalent parameter $\eta$}

From the first-order approximation formula (Eqs. (24a)-(24c)), an engineering equivalent parameter $\eta$ is found and the parameter can reflect the overall influence of the thermal conductivities of the matrix and fibers, and the interfacial characteristic on the effective thermal conductivity of a composite. For high-order numerical 

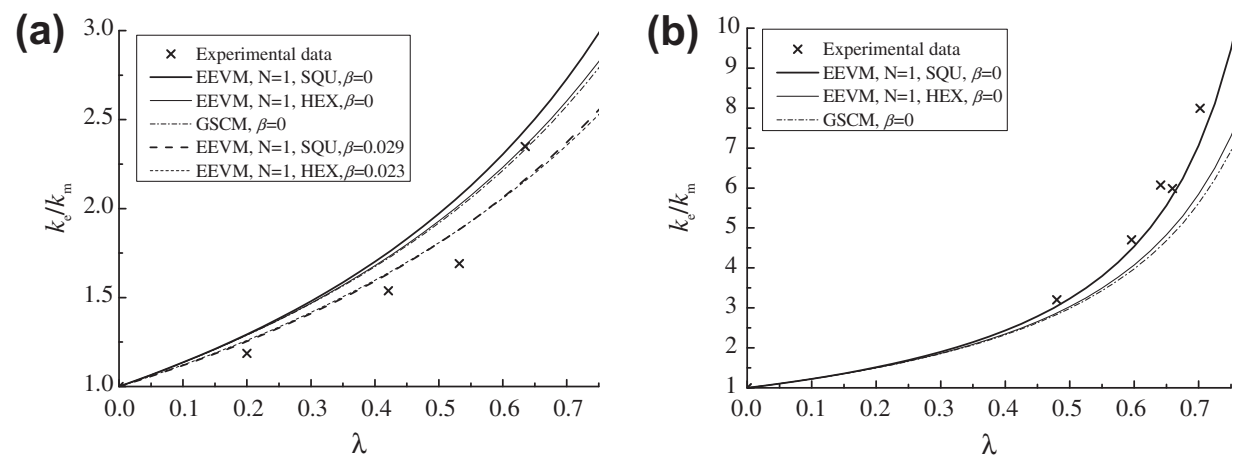

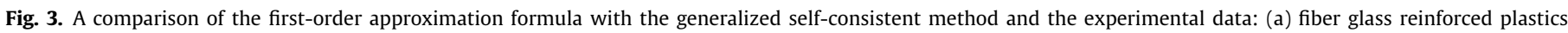
$\left(k_{\mathrm{f}} / k_{\mathrm{m}}=4.4\right)$; (b) graphite fiber reinforced plastics $\left(k_{\mathrm{f}} / k_{\mathrm{m}}=666\right)$.

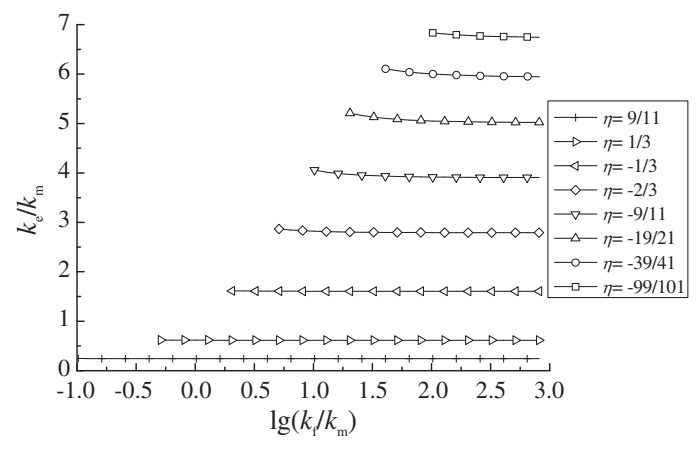

Fig. 4. The dimensionless effective thermal conductivity $k_{\mathrm{e}} / k_{\mathrm{m}}$ versus dimensionless fiber conductivity $k_{\mathrm{f}} / k_{\mathrm{m}}$ for different values of $\eta$ in the case of contact resistance and a square array of fibers, where $\lambda=0.7$.

results, does such an equivalence relation remain unchanged? To answer this problem, in the case of contact resistance, the dimensionless effective thermal conductivity $k_{\mathrm{e}} / k_{\mathrm{m}}$ versus the dimensionless fiber conductivity $k_{\mathrm{f}} / k_{\mathrm{m}}$ for different values of $\eta$ is depicted in Fig. 4, where $k_{\mathrm{f}} / k_{\mathrm{m}}>(1-\eta) /(1+\eta)$ to ensure $\beta>0$; and in the case of coated fibers, the dimensionless effective thermal conductivity $k_{\mathrm{e}} / k_{\mathrm{m}}$ versus the dimensionless fiber conductivity $k_{\mathrm{f}} / k_{\mathrm{m}}$ for different values of $\eta$ is depicted in Fig. 5a for the relative coating thickness $\xi=0.1$ and in Fig. $5 \mathrm{~b}$ for $\xi=0.01$, respectively.

From Figs. 4 and 5, it is seen that for a fixed $\eta$ and various combinations of the matrix and fibers conductivities and interfacial characteristic parameter, the dimensionless effective thermal conductivity $k_{\mathrm{e}} / k_{\mathrm{m}}$ approximately remains unchanged. The fact indicates that $\eta$ can indeed reflect a combined effect of the matrix and fiber conductivities and interfacial characteristic parameter on $k_{\mathrm{e}} / k_{\mathrm{m}}$ with a good engineering accuracy and can greatly simplify the relation of $k_{\mathrm{e}} / k_{\mathrm{m}}$ to the internal structure of a composite.

\section{Conclusions}

For a unit cell of doubly-periodic fiber reinforced composites considering the interfacial characteristics, an eigenfunction expansion of the complex potential is derived, and the expansion satisfies the governing equations of the steady-state heat conduction and different interfacial characteristics. Then using a generalized variational functional which absorbs the quasi-periodicity of the temperature field and the periodicity of the heat flux fields by the Lagrangian multiplier method, an eigenfunction expansionvariational method based on a unit cell is developed. A convergence analysis and a comparison with available experimental data and micromechanical estimations are made.

An engineering equivalent parameter $\eta$ is found and the parameter can reflect the overall influence of the thermal conductivities of the matrix and fibers and the interfacial characteristics on the effective thermal conductivity of the composite with a good engineering accuracy. The equivalent parameter greatly simplifies the complicated relation of the effective thermal conductivity to the internal structure of a composite.

By using the equivalent parameter $\eta$, the present solutions provide a unified first-order approximation formula of the effective thermal conductivity for different interfacial characteristics (perfect and thermal resistance interfaces and coating) and for the square and hexagonal arrays of fibers. A comparison with high-order numerical solutions shows that the unified first-order approximation formula possesses good engineering accuracy and can serve as a good engineering closed-form formula.
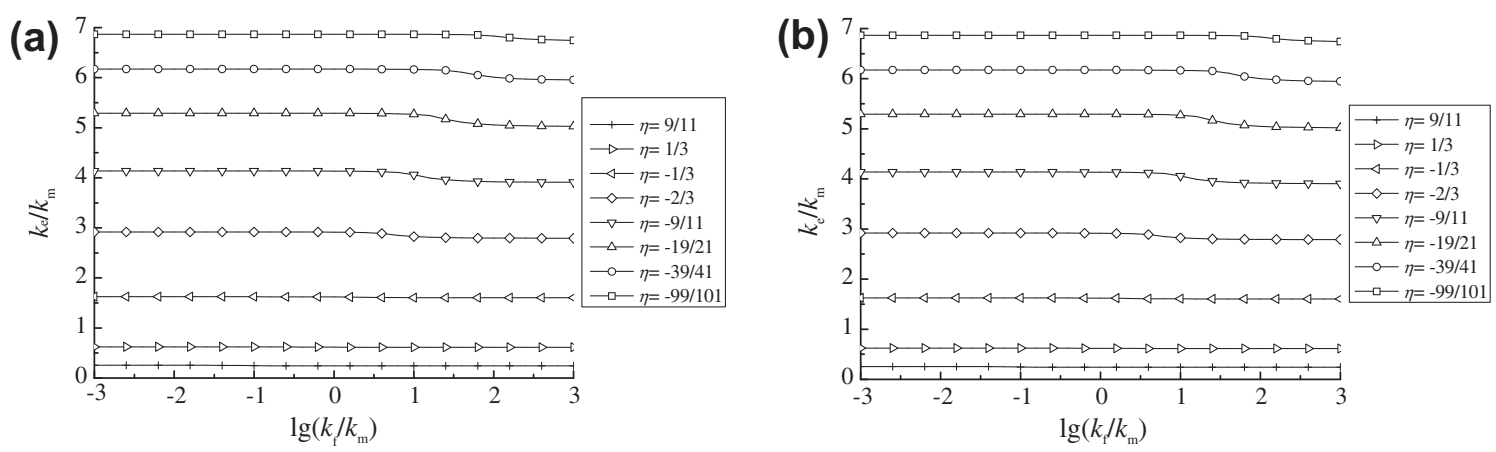

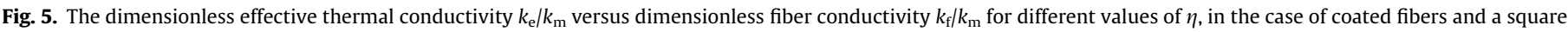
array of coated fibers $\lambda=0.7$ : (a) relative coating thickness $\xi=0.1$; (b) $\xi=0.01$. 


\section{Acknowledgements}

The work is supported by the National Natural Science Foundation of China under Grant NNSFC 90716002, and The Open Fund of LNM.

\section{References}

[1] Bhatt H, Donaldson KY, Hasselman DPH, Bhatt RT. Role of the interfacial thermal barrier in the effective thermal diffusivity/conductivity of SiC-fiberreinforced reaction-bonded silicon nitride. J Am Ceram Soc 1990;73(2):312-6.

[2] Bhatt H, Donaldson KY, Hasselman DPH, Bhatt RT. Effect of HIPing on the effective thermal conductivity/diffusivity and the interfacial thermal conductance of uniaxial SiC fibre-reinforced RBSN. J Mater Sci 1992;27(24):6653-61.

[3] Shimoda K, Park J-S, Hinoki T, Kohyama A. Influence of pyrolytic carbon interface thickness on microstructure and mechanical properties of $\mathrm{SiC} / \mathrm{SiC}$ composites by NITE process. Compos Sci Technol 2008;68(1):98-105.

[4] Hasselman DPH, Johnson LF. Effective thermal conductivity of composites with interfacial thermal barrier resistance. J Compos Mater 1987;21(6):508-15.

[5] Benveniste Y. Effective thermal conductivity of composites with a thermal contact resistance between the constituents: nondilute case. J Appl Phys 1987;61(8):2840-3.

[6] Benveniste Y, Chen T, Dvorak GJ. The effective thermal conductivity of composites reinforced by coated cylindrically orthotropic fibers. J Appl Phys 1990;67(6):2878-84.

[7] Lee YM, Yang RB, Gau SS. A generalized self-consistent method for calculation of effective thermal conductivity of composites with interfacial contact conductance. Int Commun Heat Mass 2006;33(2):142-50.
[8] Böhm HJ, Nogales S. Mori-Tanaka models for the thermal conductivity of composites with interfacial resistance and particle size distributions. Compos Sci Technol 2008;68(5):1181-7.

[9] Zou MQ Yu BM, Zhang DM. An analytical solution for transverse thermal conductivities of unidirectional fibre composites with thermal barrier. J Phys D: Appl Phys 2002;35(15):1867-74.

[10] Sun W, Lin F, Hu X. Computer-aided design and modeling of composite unit cells. Compos Sci Technol 2001;61:289-99.

[11] Gu GQ Liu ZR. Effects of contact resistance on thermal conductivity of composite media with a periodic structure. J Phys D: Appl Phys 1992;25(2):249-55.

[12] Lu S-Y, Lin H-C. Effect of interfacial characteristics on effective conductivities of isotropic two-dimensional periodic composites. Chem Eng Sci 1995;50(16):2611-31.

[13] Rolfes R, Hammerschmidt U. Transverse thermal conductivity of CFRP laminates: a numerical and experimental validation of approximation formulae. Compos Sci Technol 1995;54(1):45-54.

[14] Islam MR, Pramila A. Thermal conductivity of fiber reinforced composites by the FEM. J Compos Mater 1999;33(18):1699-715.

[15] Yan P, Jiang CP, Song F, Xu XH. Estimation of the transverse thermal conductivity of doubly-periodic fiber reinforced composites. Chin J Aeronaut 2010;23(1):54-60.

[16] Incropera FP, Dewitt DP, Bergman TL, Lavine AS. Fundamentals of heat and mass transfer. 6th ed. New York: John Wiley \& Sons; 2007.

[17] Lu S-Y. Critical interfacial characteristics for effective conductivities of isotropic composites containing spherical inclusions. J Appl Phys 1995;77(10):5215-9.

[18] Drygas P, Mityushev V. Effective conductivity of unidirectional cylinders with interfacial resistance. Quart J Mech Appl Math 2009;62(3):235-62.

[19] Thornburg JD, Pears CD. Prediction of the thermal conductivity of filled and reinforced plastics. ASME paper, 65-WA/HT-4; 1965. 